\title{
An Application of Ultrasonic Tomographic Imaging to Study Smoldering Combustion
}

\author{
STEPHEN D. TSE, ${ }^{\dagger}$ RALPH A. ANTHENIEN, and \\ A. CARLOS FERNANDEZ-PELLO* \\ Department of Mechanical Engineering, University of California, Berkeley, CA 94720, USA \\ KENJI MIYASAKA \\ Department of Technology, College of Education, Fukui University, Fukui 910, Japan
}

\begin{abstract}
An ultrasonic imaging technique has been developed and applied to examine smoldering combustion within a permeable medium. The technique provides information about local permeability variations within a smoldering sample, which can, in turn, be interpreted to visualize the propagation of the smolder reaction. The method utilizes the observation that transmission of an ultrasonic signal through a porous material increases with increasing permeability. Since a propagating smolder reaction leaves behind a char that is higher in permeability than the original (unburnt) material, ultrasonic transmission can be employed to monitor smolder progress. Additionally, the technique allows observation of the evolution of the char (i.e., material left by the smolder reaction), which, in certain circumstances, can continue to increase in permeability, due to secondary reactions (either oxidative or pyrolytic in nature). Experiments have been conducted, applying the technique to smoldering combustion in a two-dimensional geometry with line-of-sight imaging. For axisymmetric configurations, tomographic techniques have been implemented, providing three-dimensional mappings of the smolder front, as well as visualization of the smolder process itself. The results have furthered the understanding of two-dimensional smolder, and have been especially informative in identifying the controlling mechanisms leading to the transition from smoldering to flaming combustion. (C) 1998 by The Combustion Institute
\end{abstract}

\section{NOMENCLATURE}

$A \quad$ proportionality constant [1]

$c_{0} \quad$ sound speed $\left[\mathrm{LT}^{-1}\right]$

$d_{p} \quad$ pore diameter $[\mathrm{L}]$

$d s \quad$ differential path element [L]

$\Delta x \quad$ sample thickness [L]

$f \quad$ frequency $(\mathrm{Hz})\left[\mathrm{T}^{-1}\right]$

$f_{c} \quad$ characteristic frequency $(\mathrm{Hz})\left[\mathrm{T}^{-1}\right]$

$g(r)$ radial distribution function

I ultrasonic intensity $\left[\mathrm{V}^{2}\right]$

$l \quad$ metric across sample [L]

$L \quad$ sample thickness [L]

$p(l)$ projection function

$P \quad$ pressure $\left[\mathrm{ML}^{-1} \mathrm{~T}^{-2}\right]$

$\operatorname{Pr} \quad$ Prandtl number [1]

$Q \quad$ attenuation of sound wave per cycle [1]

$r$ radius [L]

$T$ temperature $[\Theta]$

$u$ average flow (Darcy) velocity $\left[\mathrm{LT}^{-1}\right]$

$V \quad$ RMS voltage [V]

$V_{O} \quad$ unattenuated signal RMS voltage [V]

*Corresponding author.

${ }^{\dagger}$ Current address: Department of Mechanical and Aerospace Engineering, Princeton University, Princeton, NJ 08544.
$V_{\varphi} \quad$ phase velocity of sound wave [ $\left.\mathrm{LT}^{-1}\right]$

$x \quad$ projection position within sample [L]

\section{Greek Symbols}

$\alpha \quad$ attenuation coefficient $\left[\mathrm{L}^{-1}\right]$

$\gamma \quad$ ratio of specific heats [1]

$\Lambda \quad$ viscous characteristic length [L]

$\Lambda^{\prime} \quad$ thermal characteristic length [L]

$\delta \quad$ viscous skin depth [L]

$\phi \quad$ porosity [1]

$\kappa \quad$ permeability $\left[\mathrm{L}^{2}\right]$

$\mu \quad$ dynamic viscosity $\left[\mathrm{ML}^{-1} \mathrm{~T}^{-1}\right]$

$\nu \quad$ kinematic viscosity $\left[\mathrm{L}^{2} \mathrm{~T}^{-1}\right]$

$\sigma \quad$ shape factor for viscous characteristic length [1]

$\sigma^{\prime} \quad$ shape factor for thermal characteristic length [1]

$\tau \quad$ tortuosity [1]

\section{INTRODUCTION}

Combustion in porous combustible materials has been investigated for several decades. The subject encompasses an extensive area, ranging from fire development in permeable solid com- 
bustibles to high-temperature synthesis of materials. Additional topics include packed-bed incineration of solid waste, in situ coal gasification, and fixed-bed catalytic reactors. The type of combustion of interest here is smoldering, which is defined as a nonflaming, self-propagating, exothermic, surface reaction, deriving its principal heat from heterogeneous oxidation of the fuel [1]. If the material is sufficiently permeable, smoldering is not confined to its outer surface and can propagate as a reaction wave through the material. Many materials can sustain smoldering, including coal, cotton, plastics, wood, tobacco, thermal and electrical insulation, and various dusts.

Smoldering combustion of porous materials has been studied both experimentally and theoretically, usually in the context of fire safety. Smoldering constitutes a serious fire risk for many reasons. It typically yields a substantially higher conversion of fuel to toxic compounds than does flaming (i.e., fast, exothermic, gasphase reactions), is difficult to detect (and extinguish) within the interior of a porous material, and provides a pathway to flaming that can be initiated by heat sources much too weak to cause a flame directly [2]. In general, porous materials do not lend themselves to visual data acquisition methods; consequently, invasive methods, such as thermocouple probing and gas sampling, are the norm. This factor has perhaps contributed to a body of smolder research, which has concentrated heavily on parametric experimental evaluations of specific materials, configurations, or limited environmental conditions.

In this work, an ultrasonic imaging technique has been applied to determine the local permeability within a smoldering material (i.e., flexible, open-cell, polyurethane foam). The permeability of a porous medium is a measure of its resistance to fluid flow through it - with low permeability associated with high flow resistance and vice versa - and can be defined on a local macroscopic scale for a porous medium continuum. Being independent of the nature of the fluid and its flow, permeability is only a function of the local pore structure geometry of the medium. With regard to this parameter, the ultrasonic imaging technique applied here exploits the physical evidence that, for an ultra- sonic frequency defined by the porous medium properties, the degree of attenuation of an ultrasonic signal through a porous material decreases with increasing permeability. Torero et al. [3] have shown that the permeability of the char left behind by the passage of a smolder wave can be up to four orders of magnitude larger than that of the virgin foam ahead of the reaction. Thus, since a propagating smolder reaction leaves behind char with a pore structure that is higher in permeability than the original material, the relative attenuation of ultrasonic transmissions can be employed to differentiate virgin (unburnt) material from char. Furthermore, the technique can document subsequent permeability developments within the char region, due to secondary reaction fronts (that also heterogeneously consume solid pore structural elements, increasing local permeability) which are not directly associated with the primary smolder front.

The images obtained with the technique not only visualize the smolder phenomenon itself (providing comparisons with temperature and species data), but also help to verify and, to a degree, quantify the important role of these aforementioned secondary reactions within the char region. These reactions form voids which provide increased permeability conditions involved in the transition from smoldering to flaming [4]. Although there exist large bodies of research dedicated to ultrasonic imaging of objects and to acoustic propagation in porous media, the application of ultrasonic imaging to smoldering combustion is truly novel and represents an important step forward in the study of this phenomenon, with potential applications in studies of other heterogeneous combustion processes.

Other methods of imaging and tomography, such as X-ray [5, 6] and gamma-ray [7, 8] have also been implemented to noninvasively examine the interior of objects. Recently, $\mathrm{x}$-ray transmission has been employed to study smoldering combustion in rigid polyurethane foam [9]. Although the most common application of these methods has been in the medical field, ultrasonic techniques have begun to find increased favor among the medical community due to the reduced health risks [10]. Several authors discuss the utilization of ultrasound as a method of 
visualizing the interior of the human anatomy $[10,11]$. Ultrasonic imaging and tomography have also been employed in many other fields [12]; for example, materials science and metallurgy utilize ultrasound for detecting structural defects [13, 14]. Despite some loss in resolution and difficulty in signal processing in comparison with other techniques such as x-ray imaging [15], ultrasonic imaging becomes the technique of choice for many applications, due to its cost-effectiveness, portability, adaptability, ease of operation, and health-risk safety.

\section{BACKGROUND}

\section{Acoustic Propagation in Porous Media}

The propagation characteristics (e.g., attenuation) of sound waves through a material medium are determined by the nature and structure of that medium. Biot's theory [16-18] shows that acoustic propagation in a fluidsaturated, porous-solid medium depends on several macroscopic parameters (i.e., porosity, elastic constants, densities of the phases, fluid viscosity, and tortuosity and permeability of the material). In the present setup, the medium is a solid fuel matrix, namely, open-cell, polyurethane foam, with an interconnected void, saturated with gaseous fluids, such as air and combustion/pyrolysis products.

For a plane harmonic sound wave, absorption is characterized by an exponential decrease in intensity of the wave with traveling distance [19]:

$I(x)=I_{0} e^{-2 \int_{0}^{x} \alpha d s}$.

Symbols are defined in the nomenclature. The attenuation coefficient, $\alpha$, of a porous medium depends on the thermophysical properties of the medium and the sound frequency, $f$.

In a viscous fluid, fluid motion due to shear surface oscillations (e.g., oscillations on the pore-scale of the local solid strands of polymer in flexible polyurethane foam in a direction parallel to the local solid/fluid interface) decays over the viscous skin depth, $\delta=(2 \cdot \nu / f)^{1 / 2}$ [20-22] (see Stokes's Second Problem [23]). Solving for the frequency when $\delta$ is of the same order as the typical pore diameter, $d_{p} \sim$

\section{TABLE 1}

Typical Properties of Flexible Polyurethane Foam

\begin{tabular}{ll}
\hline Property & \multicolumn{1}{c}{ Value } \\
\hline Porosity $\phi$ & 0.97 \\
Permeability $K$ & $2.76 \times 10^{-9} \mathrm{~m}^{2}$ \\
Density of solid $\rho_{s}$ & $1150 \mathrm{~kg} / \mathrm{m}^{3}$ \\
Specific heat of solid $c_{s}$ & $1.7 \mathrm{~kJ} /(\mathrm{kg} \mathrm{K})$ \\
Density of foam $\rho_{\text {foam }}$ & $26.5 \mathrm{~kg} / \mathrm{m}^{3}$ \\
Effective conductivity of foam $k_{\text {eff }}$ & $0.047 \mathrm{~W} /(\mathrm{m} \mathrm{K})$ \\
Heat of combustion of foam $Q$ & $3600 \mathrm{~kJ} / \mathrm{kg}$ \\
\hline
\end{tabular}

$(\kappa / \phi)^{1 / 2}$, the characteristic frequency of the fluid-saturated, porous-solid medium is obtained [20]:

$f_{c}=\frac{2 \cdot \nu \cdot \phi}{\kappa}$.

For frequencies below $f_{c}, \delta$ is larger than the typical pore size and all of the fluid within the local pores is involved in the local solid oscillation; for frequencies above $f_{c}$, only the fluid within a thickness $\delta$ of the local solid is involved [24]. Furthermore, above the characteristic frequency, the shape of the pores must be taken into consideration; whereas below it, the porosity and permeability of the medium adequately describe the geometry.

In our experiments, $f_{c} \sim 10 \mathrm{kHz}$ for the virgin polyurethane foam, (see Table 1 for material properties); and $f_{c} \sim 40 \mathrm{~Hz}$ for the char remaining behind a vigorous smolder reaction. The operating frequency of $40 \mathrm{kHz}$, selected in the present application, provides adequate spatial resolution, as well as reasonable transmittance through virgin foam (for the sample sizes of interest and ultrasonic power-output constraints). As the $40 \mathrm{kHz}$ frequency ensures that we are always in the high-frequency limit, only theory relative to this limit will be discussed.

The viscous skin depth has been related to the attenuation per cycle by Johnson et al. [25]:

$\lim _{f \rightarrow \infty} \frac{1}{Q}=\delta\left(\frac{1}{\Lambda}+\frac{\gamma-1}{\operatorname{Pr}^{1 / 2} \cdot \Lambda^{\prime}}\right)$,

with the attenuation per cycle given by [20]:

$Q=\frac{1}{2} \frac{f}{\alpha \cdot V_{\varphi}}$. 
The viscous and thermal characteristic lengths are given, respectively, by [25]:

$$
\begin{aligned}
& \Lambda=\frac{1}{\sigma}\left(\frac{8 \cdot \tau \cdot \kappa}{\phi}\right)^{1 / 2}, \\
& \Lambda^{\prime}=\sigma \sigma^{\prime} \Lambda,
\end{aligned}
$$

where $\sigma$ and $\sigma^{\prime}$ are shape factors which lie between 0.3 and 3 for most materials [25]. For cylindrical pores of circular cross section, $\sigma=\sigma^{\prime}$ $=1$.

Utilizing the above equations as well as the relation for the phase velocity in the highfrequency limit [20]:

$\lim _{f \rightarrow \infty} V_{\varphi}=\frac{c_{0}}{\tau^{1 / 2}}$,

we arrive at the following relation for the attenuation coefficient:

$$
\alpha=\frac{1}{4 c_{0}}\left(\frac{f \cdot \nu \cdot \phi}{\kappa}\right)^{1 / 2}\left(\sigma+\frac{\gamma-1}{\sigma^{\prime} \cdot \operatorname{Pr}^{1 / 2}}\right) .
$$

Thus, by measuring the attenuation coefficient, the permeability of the material can be determined using Eq. 8.

\section{Variable Properties Considerations}

Smoldering is a dynamic process characterized by many changing thermophysical and thermochemical properties. In this work, a primary aim is to relate permeability to ultrasonic attenuation. However, inspection of Eq. 8 reveals that there are several other parameters which can influence the attenuation of an ultrasonic transmission. Nonetheless, if the attenuation coefficient is a weak function of these parameters (compared to permeability), we can treat the attenuation coefficient as only a function of permeability. This section examines the relative contribution of these parameters.

\section{Porosity}

The porosity $\phi$ (or void fraction) of a medium is defined as the fraction of the total volume of the medium that is occupied by void space. Most works on smolder modeling assume that material porosity remains constant in time. This assumption is fairly reasonable since most ma- terials capable of smoldering within their interior are quite porous to begin with. For example, the flexible polyurethane foams used in this work have a porosity of 0.975 ; at most, only a $2.5 \%$ change in porosity is possible. Thus, it is reasonable to take $\phi$ in Eq. 8 to be constant at unity. Note however that $\alpha$ is still dependent on permeability, which itself is a strong function of porosity, i.e. $(\Delta \phi) / \phi \ll(\Delta \kappa(\phi)) / \kappa(\phi)$.

\section{Temperature}

Although temperature does not appear explicitly in Eq. 8, both $c_{0}$ and $\nu$ are functions of $T$. However, since $\nu$ typically varies as $T^{3 / 2}$ and $c_{0}$ goes as $T^{1 / 2}, \alpha$ will vary only as $T^{1 / 4}$. In addition to the attenuation coefficient's weak dependence on temperature, the absolute temperature of a smoldering polyurethane foam sample merely doubles with the passage of a smolder wave, while the permeability typically increases by more than an order of magnitude.

\section{Pressure}

The main parameter in Eq. 8 that is a function of pressure is $\nu$, which is inversely proportional to pressure, thus making $\alpha \sim P^{-1 / 2}$. Nonetheless, pressure variations are small, since the flow is incompressible. In fact, although we are in the high-frequency limit, the ultrasonic wavelength is still much larger than the typical pore diameter, ensuring that the flow is incompressible on the microscopic scale, and with respect to the smolder process itself.

\section{Gas Species}

The attenuation of an ultrasonic signal is dependent on the composition of the fluid, i.e., different gases and different mixtures of gases attenuate differently at different frequencies [26, 27]. The actual composition of the smolder products is not well known, making it difficult to determine the overall effect of different species. The products of combustion expected in smoldering have attenuation characteristics that are different from those of air. This, together with the fact that, at least for air as oxidizer, the combustion products are a small fraction of the gas mixture, suggests that the effect of gas composition would be small in comparison to the 
change in permeability. To verify this assumption, an experiment was conducted to determine the sensitivity of the system to the presence of combustion products. A nonreacting $\left(\sim 50^{\circ} \mathrm{C}\right)$ char sample was placed downstream of a foam sample undergoing opposed smolder. Despite the influx of gas product species from the smolder reaction, no apparent change in ultrasonic attenuation was noted during the test. The attenuation characteristics were found to be no different than that of an air-only-saturated sample, suggesting that the present imaging system is insensitive to the combustion products in the concentrations given off by a smolder reaction.

\section{Permeability Measurement}

\section{Line-of-Sight Average Permeability}

Rearranging Eq. 1, the negative log of the ratio of the received signal to an unattenuated signal (calibrated for free air) gives the value of the line-of-sight integral of the attenuation coefficient, i.e.:

$$
\int_{0}^{L} \alpha \cdot d s=-\frac{1}{2} \ln \left(\frac{I}{I_{0}}\right) .
$$

As discussed in the previous section, for our purposes then, the attenuation coefficient can be well approximated as being inversely proportional to the square root of permeability, i.e.:

$\alpha=\frac{A}{\kappa^{1 / 2}}$

where

$A=\frac{(f \cdot \nu \cdot \phi)^{1 / 2}}{4 c_{0}}\left(1+\frac{\gamma-1}{\operatorname{Pr}^{1 / 2}}\right)$

having used the assumption that the pores are circular cross-sectioned cylinders. The proportionality constant $A$ can be determined by substituting values in Eq. 11, or it can also be determined experimentally via calibration, as explained in a subsequent section. Therefore, by measuring the attenuation of a sound wave propagating through a porous medium, we can determine a line-of-sight average value for permeability; or taking it a step further, by using

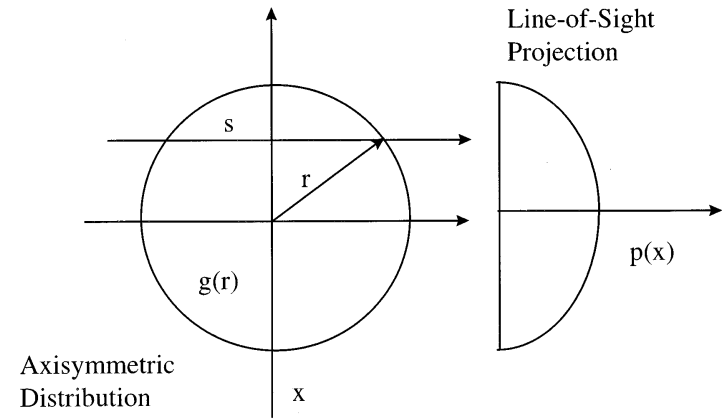

Fig. 1. Line-of-sight projection of an axisymmetric spatial distribution.

tomographic techniques, we can obtain the values of local permeability within a sample.

\section{Local Permeability via Tomography}

Tomographic methods can be used in conjunction with the ultrasonic imaging technique described above to nonintrusively monitor and image a three-dimensional smolder front, with regard to its shape, propagation, and subsequent char evolution. By deconvoluting the lineof-sight projection of a given property field, the spatial field of the measured properties can be reconstructed. The projection function $p(l)$ is related to the line-of-sight integration of the material property $g(r)$ by the following equation:

$p(l)=\int_{-\infty}^{\infty} g(r) d s$.

Figure 1 illustrates the relation between the projection and the spatial distribution of a measured property in a plane normal to the streamwise axis of an axisymmetric field.

A simple-to-implement transform, known as the Abel transform, may be used to reconstruct a radial distribution of the line-of-sight projection of an axisymmetric field [28]. The Abel transform is only one of many possible reconstruction methods available, such as the onionpeeling method and the filtered back projection method [28]. Due to its comparative ease of implementation (e.g., requiring only one set of parallel rays to be taken) and lesser computational time with respect to other methods, the Abel transform is employed in this work as a 
test of applying tomographic techniques to smoldering combustion. However, other transforms may be better suited for this type of investigation and should be explored.

The Abel transform takes the form:

$g(r)=-\frac{1}{\pi} \int_{r}^{\infty} \frac{p^{\prime}(l)}{\left(l^{2}-r^{2}\right)^{1 / 2}} d l$,

where the numerator of the integrand contains the derivative of the projection. This derivative can generate large overshoots for sudden changes in the projection function. Hughey and Santavicca [29] also indicate that the Abel transform accumulates error at the center of the distribution unless many samples are taken. This high number of samples, however, generates high-frequency noise. These effects can be minimized using the Filtered Abel Transform [28], which allows for the projection to be filtered of high wave number components prior to transformation. While filtering provides for a smoother output with less overshoot, it also reduces response. The method itself is not associated with any particular filter; nevertheless, a filter providing the best compromise of smoothness and response for the data should be chosen. Although a filtered transform has not been employed here, it is the subject of future work.

\section{IMAGING METHOD AND APPARATUS}

In the method developed here, the frequency to be employed needed to be the highest frequency (for spatial resolution purposes) that could provide a detectable transmission (at reasonable speaker output powers) through virgin foam samples of the thicknesses $(\sim 160 \mathrm{~mm})$ used throughout the present smolder study [4]. Furthermore, the relative magnitudes of transmission between virgin foam and char had to be sufficiently different such that a smoldering interface could be visualized. The $40 \mathrm{kHz}$ frequency applied in this work performed best under these criteria, with commercially available pressure transducers (speakers/microphones). The spatial resolution is of the order of $10 \mathrm{~mm}$, corresponding to the ultrasonic wavelength and the size of the diaphragm of the microphone. Only a limited number of commercial pressure transducers were tested; conceivably, pressure transducers with frequency responses higher than $40 \mathrm{kHz}$ that provide better resolution and adaptability may be available and should be investigated.

Another design consideration is whether to employ a continuous ultrasonic wave or an ultrasonic wave-train pulse. Although slightly more difficult to employ, an ultrasonic wavetrain pulse allows a more precise transmission measurement because the first peak in the received wave-form identifies the desired transmitted signal through the foam/char, distinguishing it from reflection and other interference signals. In addition, the frequency at which the wave-train pulses are sent can be reduced, permitting the speakers to be driven at higher powers without overheating.

\section{Operating Procedure}

The operating procedure and design considerations, for the ultrasonic imaging technique for a single speaker/microphone set are as follow:

1. A speaker sends a $40 \mathrm{kHz}$ ultrasonic sinusoidal wave-train pulse through the porous medium. As shown in Fig. 2, the wave-train pulse consists of a given number of cycles. The duration of the wave-train pulse is experimentally determined to maximize amplitude magnitude versus time spread of the transmitted wave-train pulse, due to superposition of diffracted and reflected signals in the porous medium.

2. A microphone receives a waveform, which includes the transmitted wave-train pulse along with reflection and other interference signals. This waveform is amplified and converted to an RMS signal. Parenthetically, we are only interested in relative magnitudes of transmission-amplitude modulation; thus the absolute value of the RMS signal is not critical. Based on the shortest path length through the sample, the first peak in the received waveform identifies the desired transmitted signal through the foam/char.

3. The received RMS waveform is digitally sampled by a computer and stored into memory. The entire received waveform constitutes a 


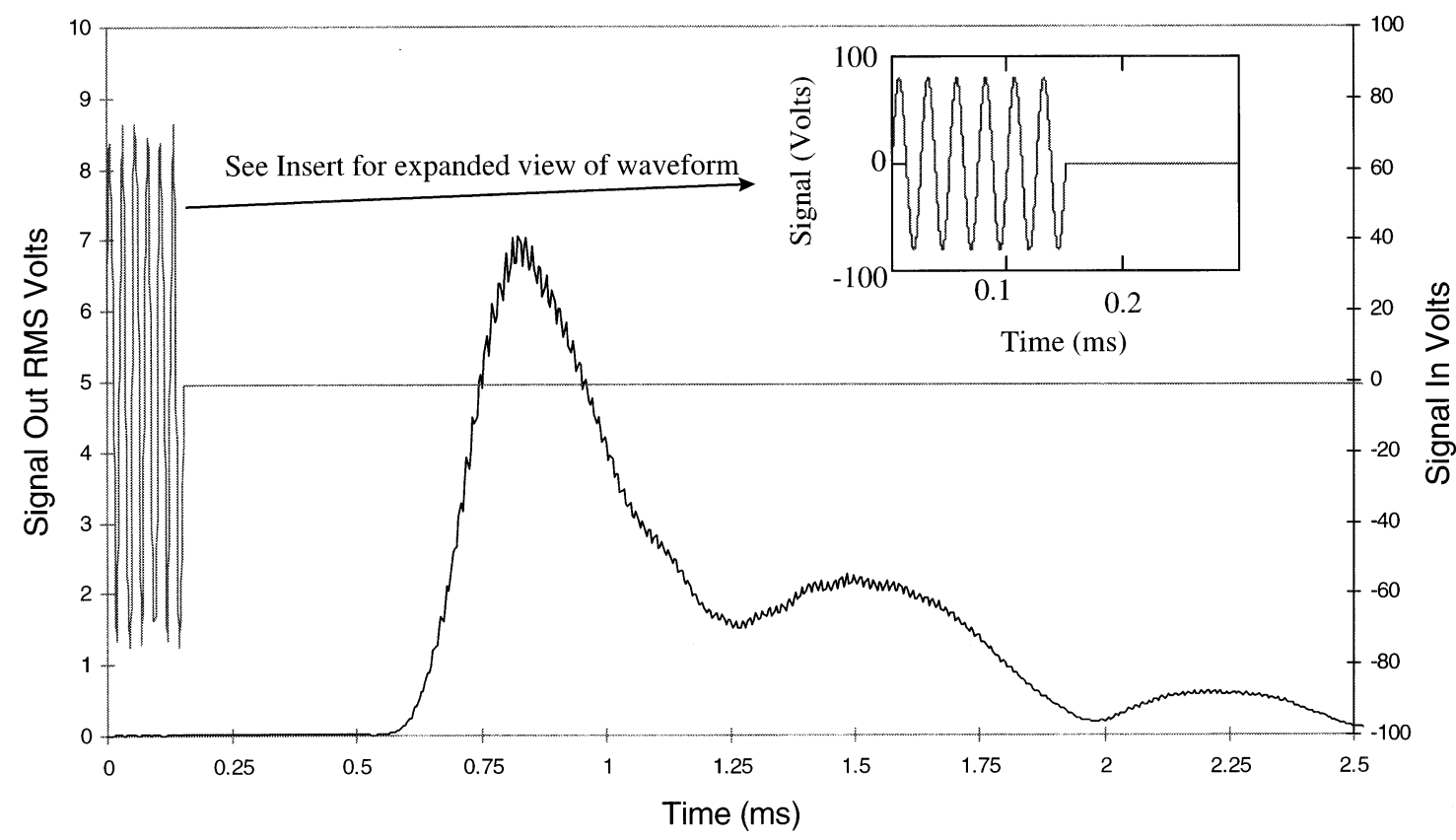

Fig. 2. Sample input and received waveforms.

single ultrasonic transmission data point, where the attenuation of the transmitted wave-train pulse is deduced in postprocessing. Figure 2 shows the waveform received by a microphone, after amplification and RMS conversion.

4. The next ultrasonic transmission data point is taken. The frequency of wave-train pulses is very important. The time between each wave-train pulse must be longer than the time-of-flight for a single pulse. Enough time must also be allowed to minimize superposition effects from previously sent wave-trains that can be reflected back into the propagation path of interest. The time interval is limited by the desired rate of ultrasonic transmission data points to be taken.

As indicated above, an entire received waveform represents a single ultrasonic transmission data point. The line-of-sight ultrasonic intensity attenuation is measured in terms of the RMS voltage ratio measured by the speakers, such that Eq. 9 can be written as:

$$
\int_{0}^{L} \alpha \cdot d s=-\ln \left(\frac{V}{V_{0}}\right),
$$

where $V_{0}$ is calibrated for attenuation due to beam expansion.

\section{Apparatus}

In the present setup, linear arrays of speaker/ microphones are employed, to reduce the time needed to scan the sample. The schematic of the ultrasonic imaging technique used in this work is shown in Fig. 3. Line-of-sight transmission projections are produced by scanning the samples with horizontal arrays of speakers and microphones, mounted to a 2-axis moving UniSlide assembly. $40 \mathrm{kHz}$ sinusoidal waves in six-period bursts are produced by a Sony/Tektronix AFG310 synthesized function generator, triggered by a Hewlett Packard 3312A function generator at $10 \mathrm{~Hz}$. The pulsed signals are amplified to $75 \mathrm{~V}$ peak-to-peak and are sent sequentially, via Opto22 ODC5R relay modules, to an array of 8 muRata MA40A3S ultrasonic speakers (16 mm diameter). An array of 8 muRata MA40A3R ultrasonic microphones (16 $\mathrm{mm}$ diameter) receive the transmitted pulsed signals. These received waveforms are preamplified, multiplexed, amplified by a gain-selectable differential amplifier, converted to a 


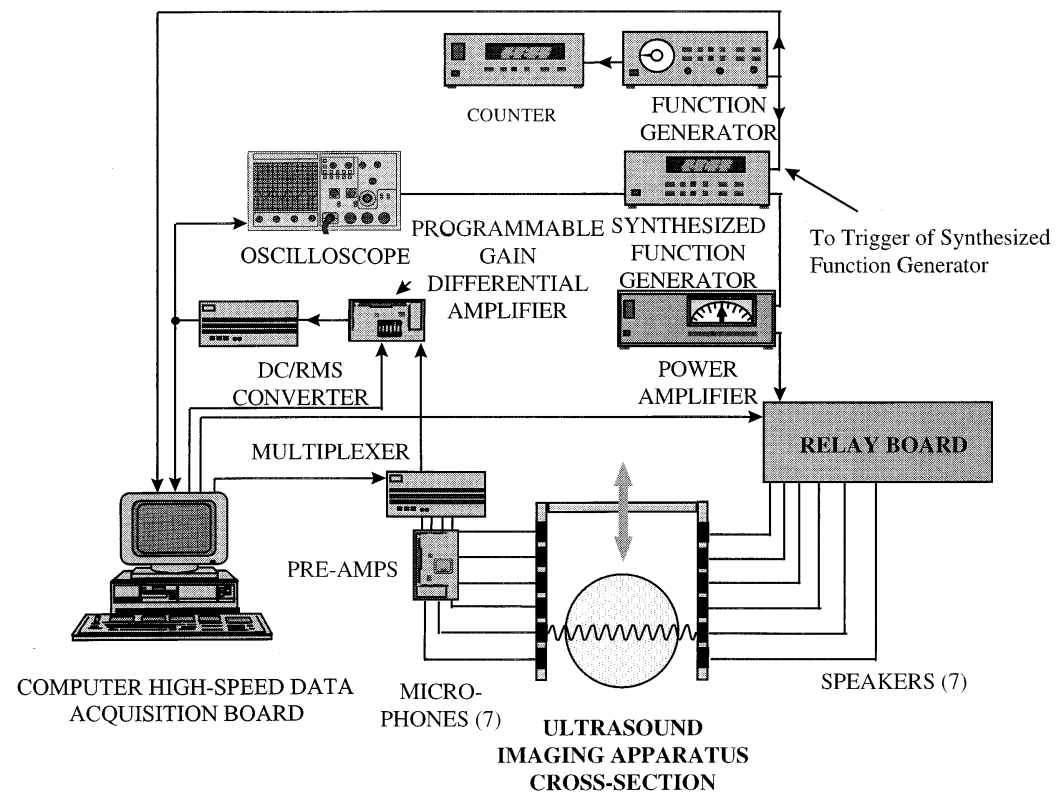

Fig. 3. Ultrasonic imaging system apparatus and setup.
RMS/DC signal, sampled at $250 \mathrm{kHz}$ by a high-speed $\mathrm{A} / \mathrm{D}$ board (which is triggered by the function generator at $10 \mathrm{~Hz}$ ), and recorded onto a personal computer.

\section{CALIBRATION TECHNIQUE}

\section{Line-of-sight Average Permeability As a Function of Ultrasonic Attenuation}

The implementation of the ultrasound technique requires calibration of the relation between permeability and ultrasonic attenuation. Assuming that attenuation of a transmitted ultrasonic signal is only a function of permeability, as described previously, the permeability calibration becomes straightforward. Inserting the expression for $\alpha$ of Eq. 10 into Eq. 14, we solve for $A$, to obtain:

$A=-\frac{\kappa^{1 / 2}}{L} \ln \left(\frac{V}{V_{0}}\right)$,

for a sample of isotropic permeability. By measuring the ultrasonic intensity attenuation for a virgin polyurethane foam sample, with isotropic permeability $\kappa_{\text {foam }}$, and sample thickness $L$, the proportionality constant $A$, which relates acoustic attenuation to permeability, can be deter- mined. Note: $\kappa_{\text {foam }}$ is predetermined experimentally via Darcy's law:

$u=-\frac{\kappa}{\mu} \frac{\Delta P}{\Delta x}$,

for an isotropic medium. In this manner, the value for the proportionality constant $A$ has been reported by Tse [30] to be $A=9.19 \times$ $10^{-4}$, which compares favorably with the value $A=8.31 \times 10^{-4}$, obtained with Eq. 11 .

Another calibration method is to apply the technique by varying the thickness of a virgin foam sample, while keeping the distance $L$, between speaker and microphone fixed. Assuming that the attenuation of a transmitted ultrasonic signal is only a function of permeability, such a calibration is possible through the use of Darcy's law (Eq. 16) because a thinner sample will appear to have a higher line-of-sight permeability with respect to a fixed distance $L$ $(L>\Delta x)$. Fixing $u, \mu$, and $\Delta P, \kappa$ becomes proportional to $\Delta x$; thus $\kappa_{\text {eff }}=\kappa_{\text {foam }} \cdot L / \Delta x$, and permeability as a function of ultrasonic attenuation can be obtained accordingly. Figure 4, which also includes data points for actual char samples, confirms the validity of Eq. 15. This approach was used to verify the results of the previous calibration technique. 


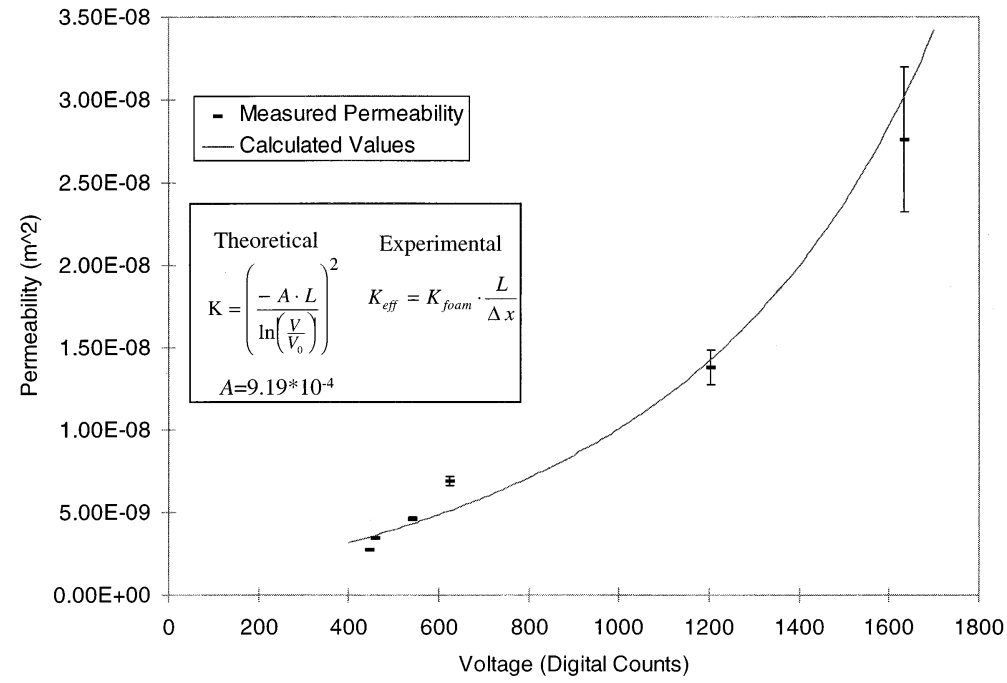

Fig. 4. Permeability calibration for ultrasonic imaging technique.

\section{Sample Shape Calibration/Filter for Tomography}

For tomography, a further needed calibration consists of a baseline scan of the sample prior to smoldering. Since the virgin sample is of uniform composition, deviations of the deconvoluted measured permeability, acquired from the baseline scan, from that of constant permeability are, in large part, attributed to factors involving the shape of the sample and the position of the speaker/microphone set with respect to the sample. For example, because of the "large" diameter of the ultrasonic "beam," measurements occurring at the edges of the sample are subjected to increased signal strength from ultrasonic transmission passing around the sample. Subsequent permeability measurements of the smoldering sample are calibrated with respect to the baseline scan.

The shape-calibration/filtration procedure is as follows. A uniform composition, cylindrical sample of flexible, polyurethane foam is scanned, and the sample-lengthwise average values of the resulting ultrasonic attenuation data for each point along the radius are calculated and stored. Deviation of the data from the mean at each radial point is also calculated and stored. In this manner, an envelope is created, against which data from the smoldering sample may be compared. In data processing, any measurement falling within this envelope is set to the value for an ideal projection; and any measurement falling outside of the envelope is shifted by the difference between the average value of the envelope and the ideal projection value at that radial point. Values for the ideal projection can be generated by performing the Radon transform [28] on a unit disk of unity property. This method of shape calibration/ filtration also accounts for variations from the ideal projection due to surface waves, diffraction of the wave, imperfections in the virgin foam, and any slight differences between our array of speaker/microphone pairs. The tradeoff of this calibration/filtering method is decreased resolution of permeability.

Shape-calibrated/filtered projections of the smoldering sample are later deconvoluted using the Abel transform to reconstruct a two-dimensional axisymmetric spatial field of the attenuation coefficient. A three-dimensional image of material permeability is then constructed from a vertical stack of the deconvoluted two-dimensional slices. Figure 5 shows the results of the shape-calibrated/filtered deconvoluted projection of a phantom sample-a polyurethane foam cylinder, with an inner cylinder of a smaller radius and lateral length composed of char. As evidenced by Fig. 5, we have determined that the tomographic technique presents an accurate picture of the scanned foam/char sample. 

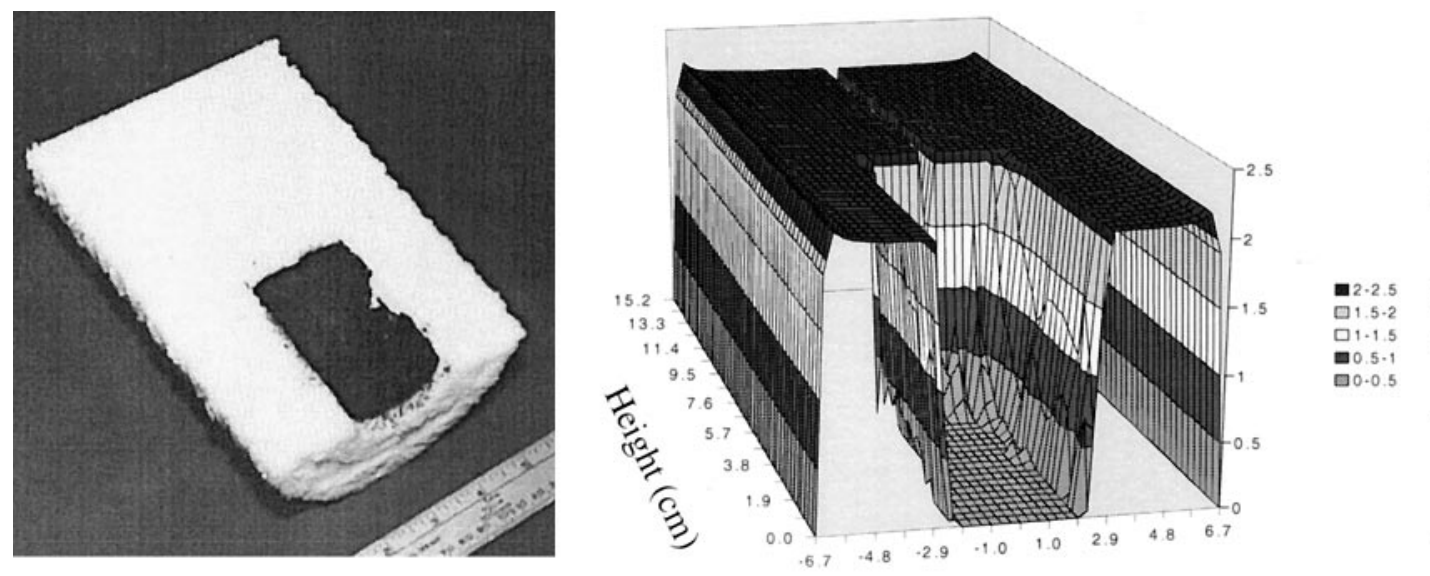

Radius (cm)

Fig. 5. Tomographic attenuation values for a cylindrical phantom as functions of $r$ and $z$. Picture of section of actual sample.

\section{APPLICATION OF ULTRASONIC IMAGING TO SMOLDERING COMBUSTION}

\section{2-D Imaging of a Smoldering Sample}

An example of the applicability of the ultrasonic imaging technique to examine smoldering combustion is shown in the study of the mechanisms leading to the transition from smoldering to flaming combustion in a two-dimensional geometry conducted by Tse et al. [4]. In this study, an average permeability is measured by the line-ofsight integral of the ultrasonic attenuation coefficient, as given in Eq. 14. A schematic of the experimental facility is shown in Fig. 6. All tests are conducted using open-cell, unretarded, flexible polyurethane foam $\left(26.5 \mathrm{~kg} / \mathrm{m}^{3}\right.$ density and 0.975 void fraction) with air as oxidizer. Smolder tests are conducted in a wind-tunnel test section $(0.6 \mathrm{~m}$ long with a rectangular cross section $0.127 \mathrm{~m}$ wide and $0.07 \mathrm{~m}$ deep). Three of the wind-tunnel walls are made of quartz for optical access; and the fourth wall is established by the exposed surface of the foam sample, mounted flush with the wind-tunnel wall. The foam sample is a rectangular parallelepiped (0.406 $\mathrm{m}$ long with a cross section $0.127 \mathrm{~m}$ by $0.14 \mathrm{~m}$ ). Three side surfaces and the top of the sample are insulated with Fiberfrax sheets. House-compressed air flows through the tunnel settling chamber and a converging nozzle before entering the test section. Smolder ignition is accomplished with an electrically heated igniter placed in contact with the foam at the sample bottom. The smolder ignition requires a supply of $70 \mathrm{~W}$ for $3000 \mathrm{~s}$, heating the igniter to about $500^{\circ} \mathrm{C}$. At that time, the heater and igniter air flow are turned off; and the external air flow is turned on and forced upward and parallel to the exposed foam surface.

Line-of-sight average permeability images of the sample, taken at two periods during its smolder, are presented in Fig. 7. Producing a single image requires approximately 10 minutes due to limitations on scanning and data storage rates. Figure 7a corresponds to a period prior to flaming when the smolder front is propagating at $29 \mathrm{~cm}$ from the igniter. Figure $7 \mathrm{~b}$ corresponds to the sample immediately after flaming; note that combustion is immediately extinguished with a nitrogen purge upon flaming. The shades of gray-scale describe the line-of-sight value of permeability, with white corresponding to virgin foam and black to air (void). The delineation of the smolder front can be clearly identified in Fig. 7. In addition, ultrasonic imaging shows that the char left behind by the smolder wave increases in permeability (via the formation of voids) as smolder progresses. It is seen that the technique provides information about the location of the smolder front and the changes in permeability throughout the char region. The presence of voids is clearly observed in Fig. 7, and further corroborated with visual observation of the char after completion of an experiment. 


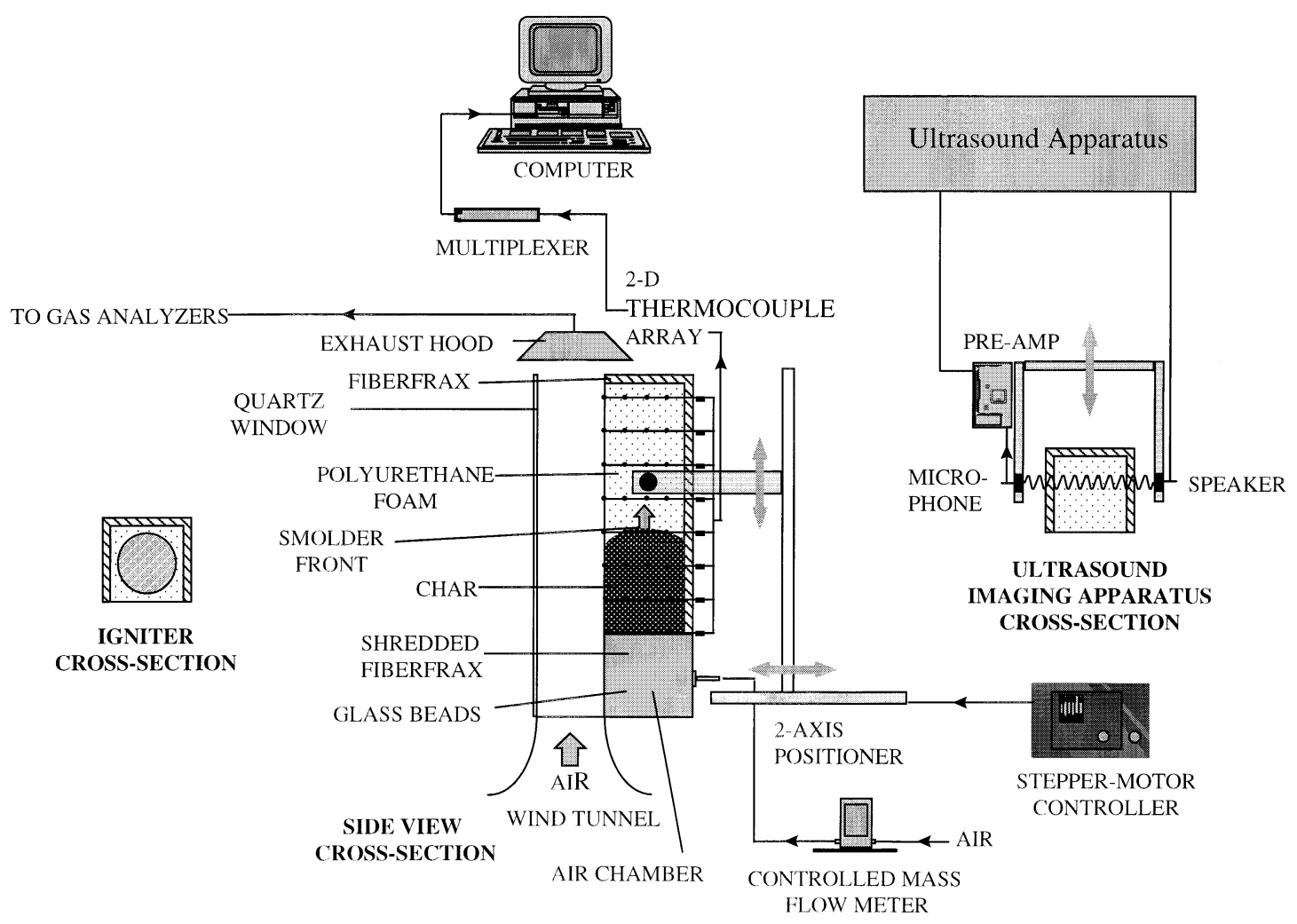

Fig. 6. Experimental setup for two-dimensional smolder experiment.

By scanning in a reduced region, the ultrasonic imaging technique can also be used to obtain the evolution of the char permeability at a particular location in the sample (along the centerline at a height of $15 \mathrm{~cm}$ ). The results presented in Fig. 8 show that the char continues to react and increase substantially in permeability long after the primary smolder front has passed, confirming that secondary char oxidation is responsible for the formation of the voids. Corresponding temperature distribution data along the length of the sample for increasing time (see Tse et al. [31]) elucidates the absence of a well-defined smolder temperature and blurring of the smolder zone, but clearly manifesting the propagating smolder front. Other experiments have also shown that the "smolder zone" in forward smolder is difficult to define, as compared to opposed smolder, and often seems thick and continuous.

Ultrasonic images, as shown in Fig. 7, together with thermocouple probing data of the foam interior and schlieren interferometry im- ages of the gas phase at the foam/air-flow interface, reveal that transition to flaming, in this case, is not the result of an accelerating smolder, but of the onset of gas-phase reactions in the void-filled, hot char region. The increasing permeability of the char, which favors the transport of oxidizer and volatiles to its interior, in conjunction with the reduced heat loss property of a self-insulating porous medium (less heat losses per unit volume of smoldering material to the surrounding tunnel walls and air at the bulk medium interface), results in the transition to flaming. Gas analyzer data of mixedaggregate exhaust gases indicate significant increases in hydrocarbon production as the smolder nears transition to flaming. The larger pore size in the char favors gas-phase reactions; in contrast, the smaller pore size $(\sim 200 \mu \mathrm{m})$ near the smolder front tends to suppress gasphase reactions by surface quenching of the free radicals, encouraging solid surface attack of oxygen [1]. In the immediate vicinity of the local char surface where the temperature is most 

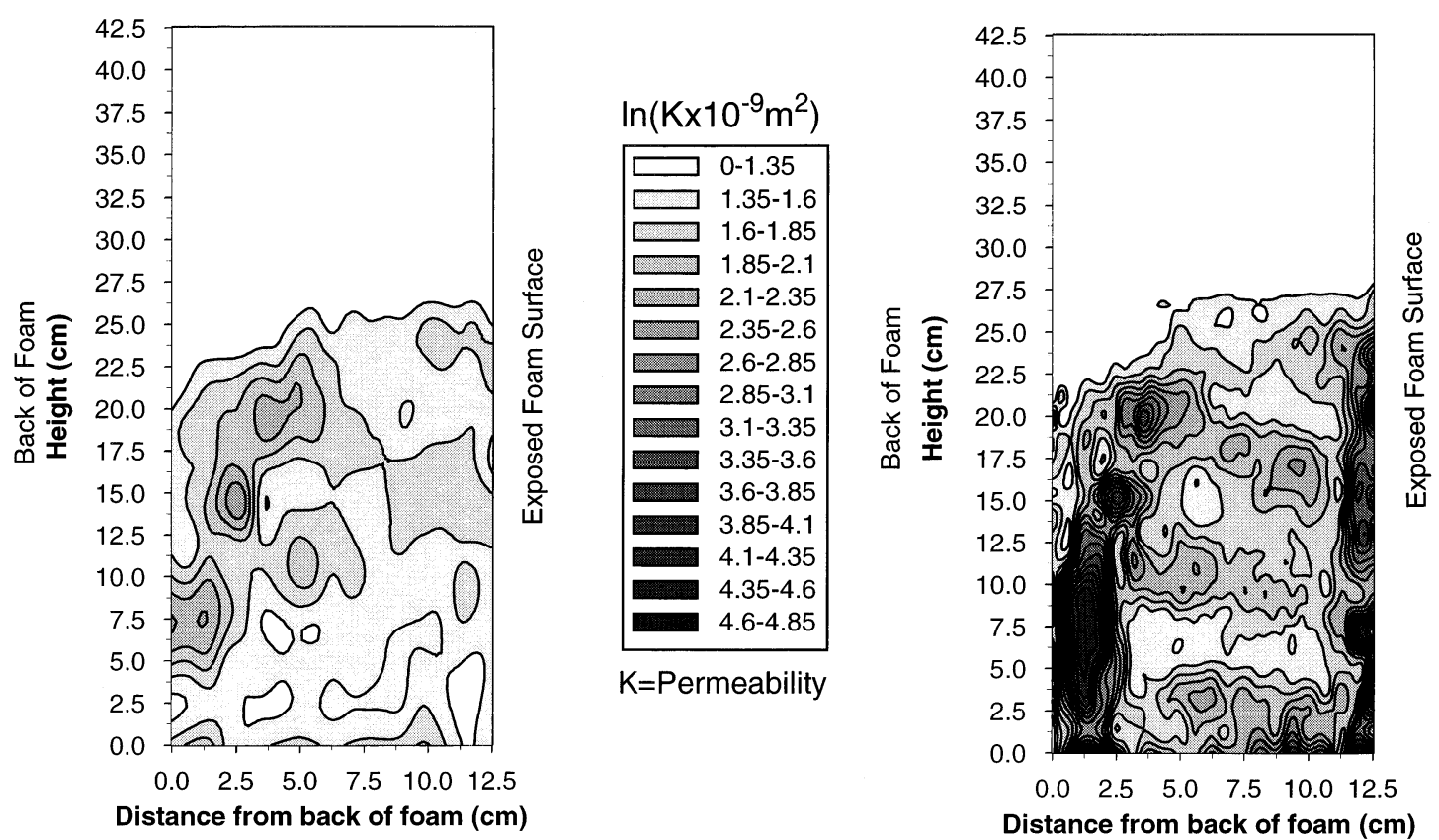

Fig. 7. Time evolution for line-of-sight average permeability of ultrasonic image of two-dimensional smoldering.

favorable for a high reaction rate, the reactant concentration is least favorable; oxygen concentration will be the lowest (compounded by the fact that surface reactions are competing with gas-phase reactions for oxidizer) and fuel and product concentrations will be the highest. The proposed sudden influx of oxidizer, allowed in by increased permeability of the char, as confirmed by ultrasonic imaging, provides the impetus needed for the transition. More detailed information about the process can be found in Tse et al. [4].

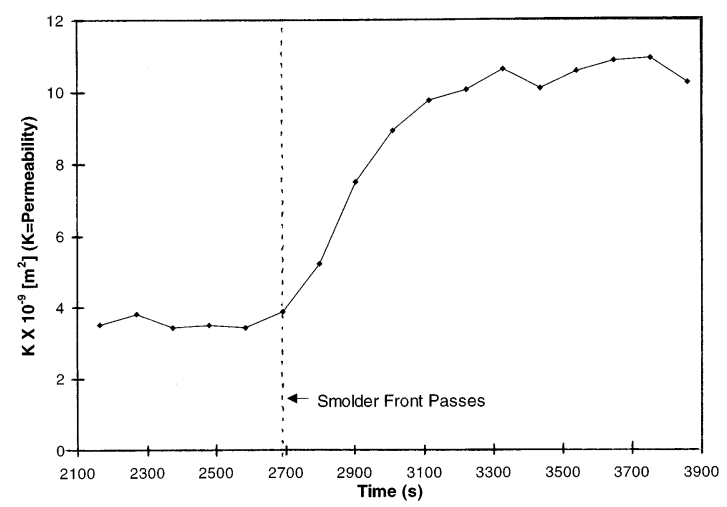

Fig. 8. Time evolution of line-of-sight average permeability in char at one location using ultrasonic imaging.

\section{Tomography of Axisymmetric Smoldering}

Ultrasonic tomography has been applied to examine axisymmetric, downward natural convection smoldering. Again, all tests are conducted using open-cell, unretarded, flexible polyurethane with air as oxidizer. A schematic of the experiment is shown in Fig. 9. The foam sample is a cylinder, of $130 \mathrm{~mm}$ diameter and $200 \mathrm{~mm}$ length, with the lateral surface of the sample left exposed. Smolder ignition is accomplished with an electrically heated igniter placed in contact with the foam at the sample top. The entire sample is caged in a wire mesh, with square openings of 12.5 -mm sides, for support. A 2-D array of thermocouples is placed within the sample, spaced at $30 \mathrm{~mm}$ radially and $20 \mathrm{~mm}$ axially. The temperature data is used to determine independently the progress of the smolder front and the strength of the reaction.

A sequence of tomographic images and the corresponding 2-D thermocouple data as the smolder reaction progresses down the sample are shown in Figs. 10a-d. Due to present limitations on scanning and data storage rate, a coarse sampling grid is employed, allowing for several complete traverses while the sample is 


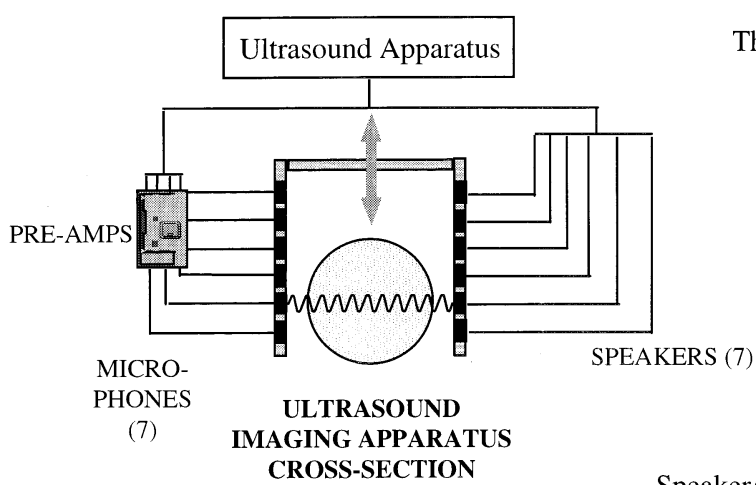

Speaker/Microphone Array

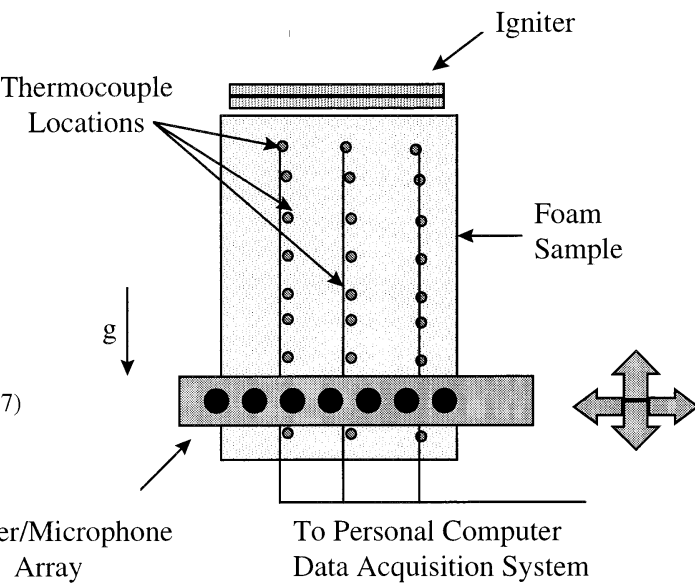

Fig. 9. Experimental setup for axisymmetric experiment using ultrasonic tomography. undergoing the smolder process. Each of Figs. $10 \mathrm{a}-\mathrm{d}$ shows a graph of permeability versus position (as measured by the ultrasonic tomographic technique), and a graph of temperature versus position (as measured by the 2 -D thermocouple array). In each graph, darker shades of gray-scale indicate greater values. Each temperature graph has been time-skewed to properly reflect the temperature at each time and position of the permeability chart. Each chart does not represent one instance in time, but rather a range of time, which corresponds to the amount of time necessary to complete one traverse of the ultrasound scanning apparatus. The smolder reaction temperature is taken to be about $350^{\circ} \mathrm{C}$, which corresponds to a dark gray in the temperature graphs.

Figures 10a-d evince that the ultrasonic imaging technique accurately tracks the progression of the smolder front though the sample via the resulting change in permeability. The temperature data taken during smoldering and shown in these figures compare extremely well with and validate the ultrasound data. Comparison of Fig. 10c and Fig. 10d reveals that the char has continued to evolve well after the smolder front has passed.

For a truly axisymmetric field of material property, only projections from 0 to $\infty$ need to be taken and deconvoluted. Note, however, that in Fig. 10, two sets of deconvoluted projections are displayed adjacently for each permeability image sequence: negative values of $r$ correspond to the deconvolution of the projection $p(l)=$ $\int_{-\infty}^{0} \alpha(r) d s$; and positive values of $r$ correspond to the deconvolution of the projection $p(l)=$ $\int_{0}^{\infty} \alpha(r) d s$. The slight differences between the two sets of permeability field in the sequences of Fig. 10, show the nonaxisymmetric nature of the actual smolder process and the need for future development of nonaxisymmetric tomographic imaging.

Reinspection of the sequence in Fig. 10 shows decreasing local permeability with increasing time after the passage of the smolder front for some locations (e.g., region around $z=14$ to 16 $\mathrm{cm}$ and $r=-2$ to $-3 \mathrm{~cm}$ ). This phenomenon is most likely due to reaction products condensing in the cooler char region behind the downward propagating (opposed flow) smolder reaction. The condensed products (tar and water) plug the pores thereby reducing the permeability. This effect has also been observed in recent forward smolder experiments.

Visual comparison of the sample with a final ultrasound image was not possible because the smolder reaction reversed upon reaching the bottom of the sample. The resulting dramatic increase in permeability exceeded the dynamic range of our system. Even so, we were able to track the upward movement of the reaction as it progressed through the sample. A future system with increased dynamic range would be able to quantify the extent of the reaction moving upward through the char, and allow for post-test visual comparison. 
(a)

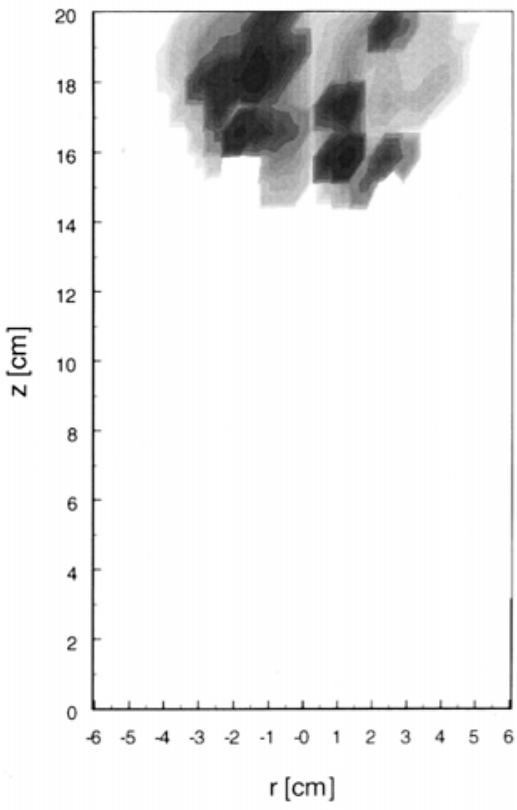

(b)

$$
\text { Permeability }
$$

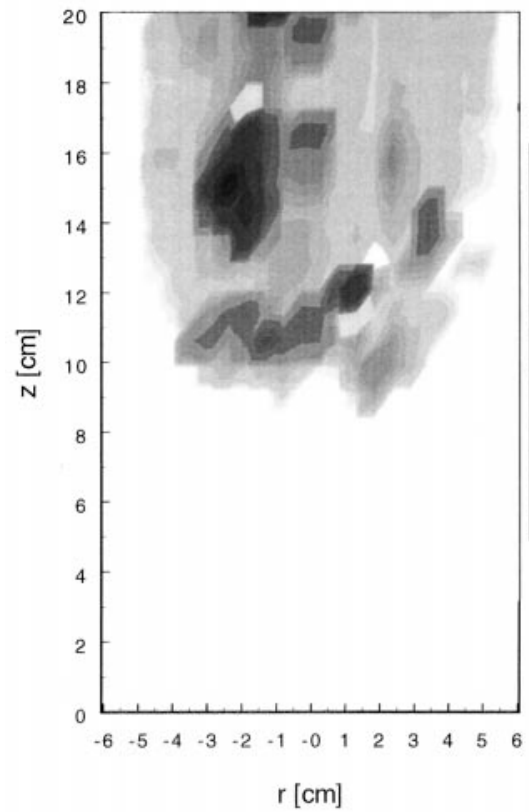

Temperature

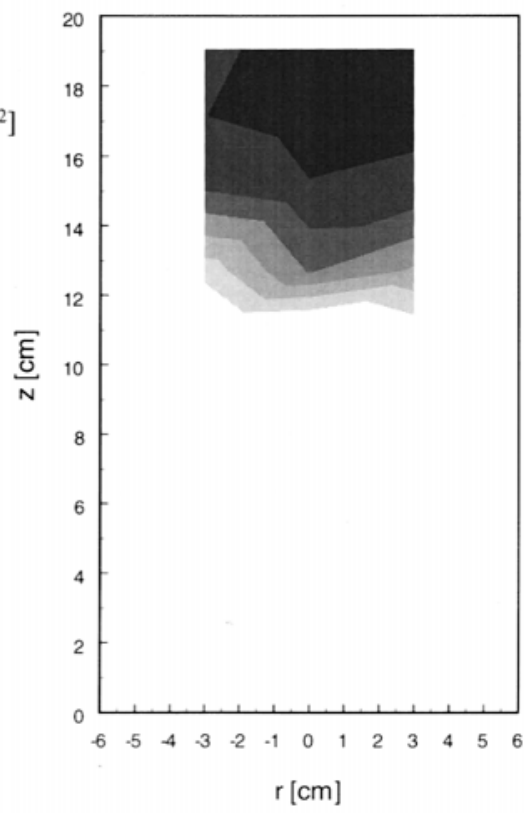

Temperature

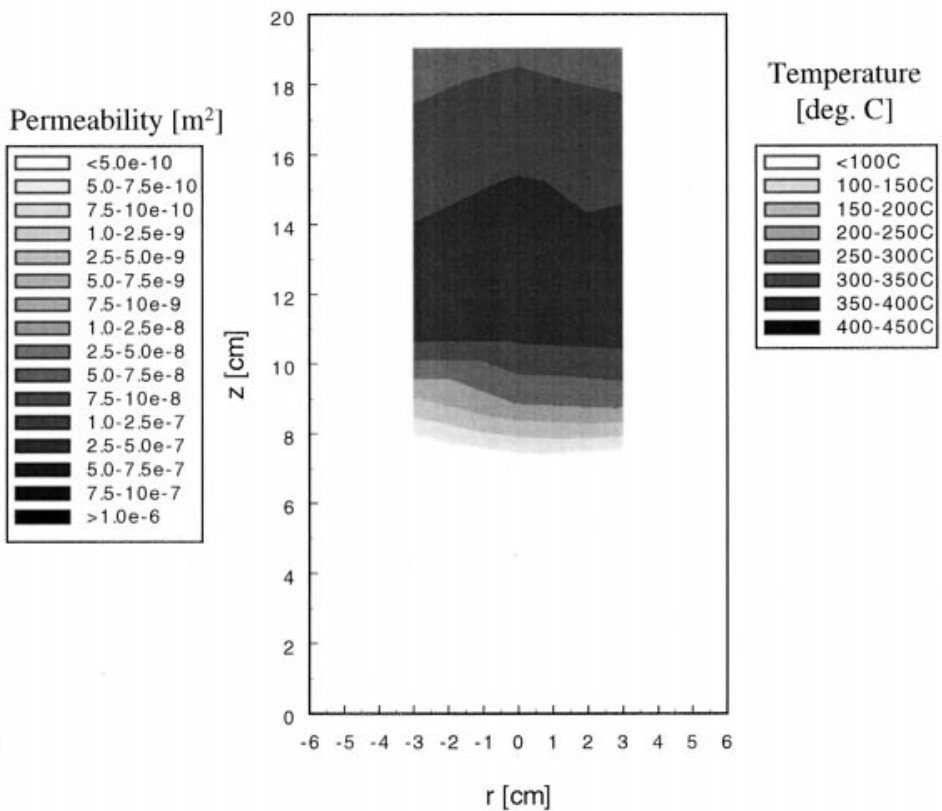

Fig. 10. Time evolution of permeability (using ultrasonic tomography) and temperature (via thermocouple probing) as functions of $r$ and $z$. (a) Shortly after ignition; (b) approximately $400 \mathrm{~s}$ after (a); (c) approximately $200 \mathrm{~s}$ after (b); (d) approximately $400 \mathrm{~s}$ after (c). 
(c)

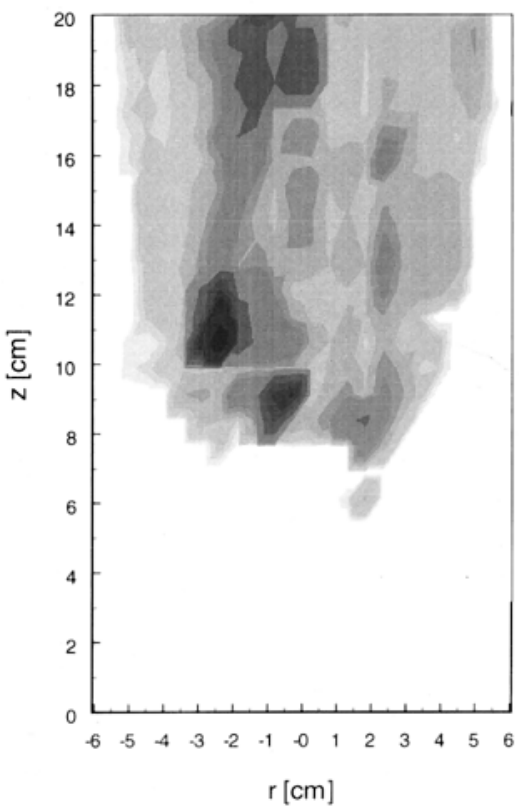

(d)

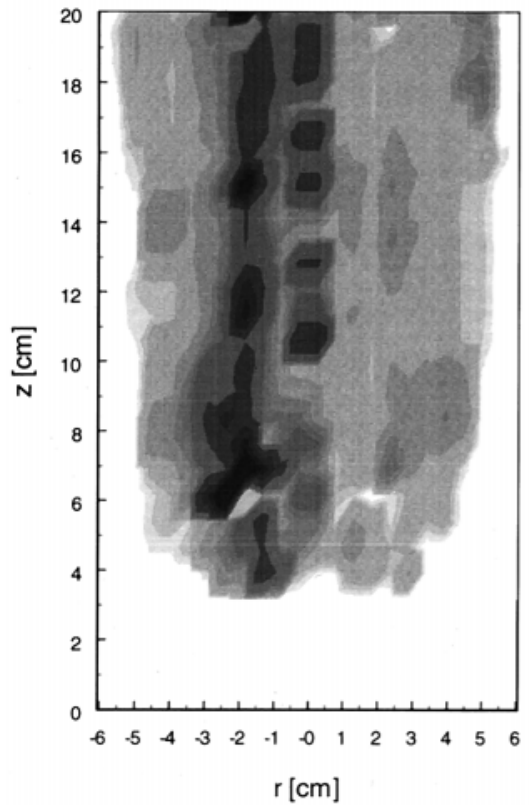

Temperature

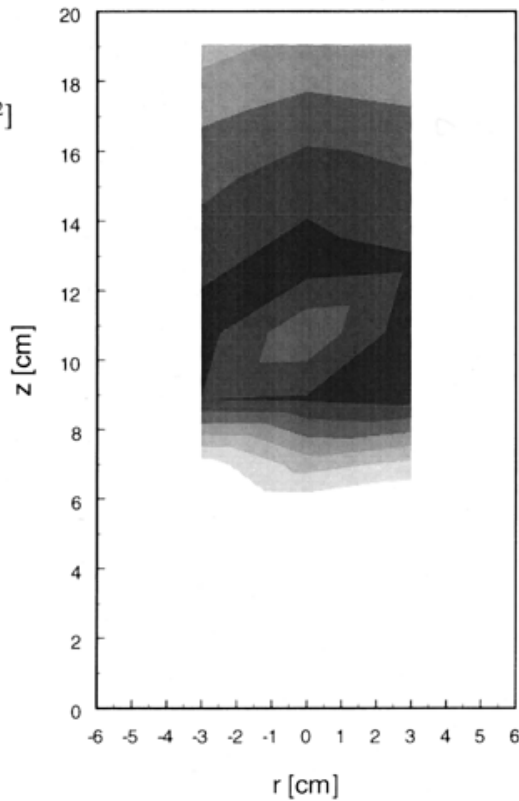

Temperature [deg. C]

\begin{tabular}{|c|c|}
\hline$\square$ & $<100 \mathrm{C}$ \\
\hline$\square$ & $100-150 \mathrm{C}$ \\
\hline$\square$ & $150-200 \mathrm{C}$ \\
\hline & $200-250 \mathrm{C}$ \\
\hline & $250-300 \mathrm{C}$ \\
\hline & $300-350 \mathrm{C}$ \\
\hline & $350-400 \mathrm{C}$ \\
\hline & $400-450 \mathrm{C}$ \\
\hline
\end{tabular}

Temperature

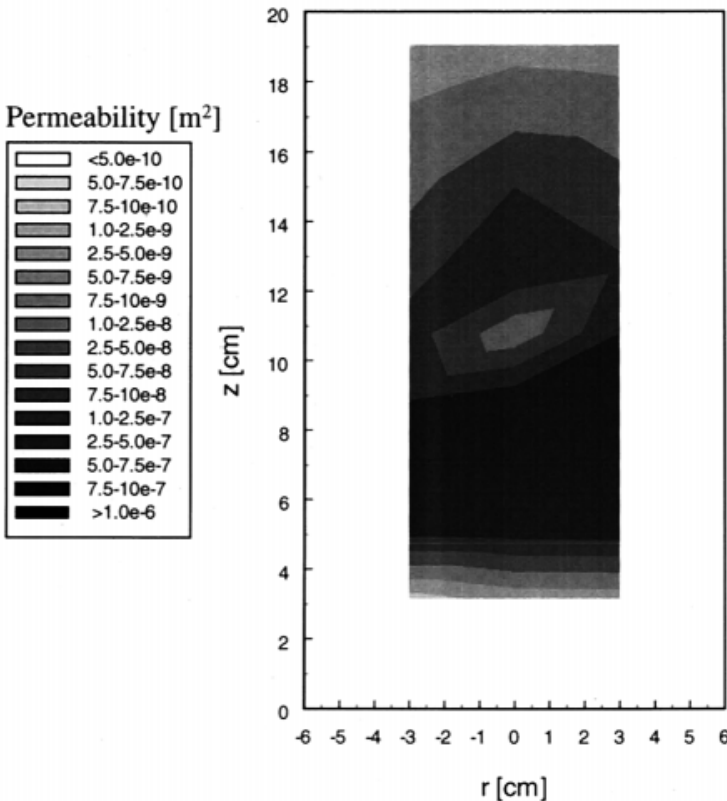

Temperature [deg. C]

Fig. 10. Continued.

\section{CONCLUDING REMARKS}

The innovative application of ultrasonic imaging as employed here brings the study of opti- cally inaccessible heterogeneous combustion into a new perspective, where new insight into the mechanisms controlling smoldering combustion is revealed via visualization of evolving 
material properties. The technique is especially informative concerning char permeability evolution and propagation of the smolder front. Future development of this technique should include ultrasonic tomography of nonaxisymmetric material permeability. Also worth pursuing is the determination of the temperature fields within a smoldering fuel. This could be accomplished by measuring the time-of-flight of the ultrasonic signal though the permeable material. As the sample temperature increases, the sound speed also increases, thereby decreasing the time of flight. A higher speed data acquisition system than the one employed in this work would be necessary due to extremely short times involved in measuring this variation.

This work is supported by the National Aeronautics and Space Administration under Grants NAG3-2026 and NGT-51268. Thanks are due to Dr. David Urban for his helpful suggestions and comments during the course of this work, and to Mr. David Walther for his assistance and suggestions. Thanks are also due to Mr. A. Uozaki of Komatsu Murata Seisakusho Co. for providing the ultrasonic transducers, and to Professor George Johnson for his advice.

\section{REFERENCES}

1. Ohlemiller, T. J., Prog. Eng. Comb. Sci. 11:277-310 (1985).

2. Ohlemiller, T. J., (1986) Smoldering combustion. NBSIR 85-3294, National Bureau of Standards.

3. Torero, J. L., Fernandez-Pello, A. C., and Kitano, M., Combust. Sci. Technol. 91:95-117 (1993).

4. Tse, S. D., Fernandez-Pello, A. C., and Miyasaka, K., Twenty-Sixth Symposium (International) on Combustion, The Combustion Institute, Pittsburgh, 1996, pp. 1505-1513.

5. Gaarder, N., and Herman, G., Comput. Graphics Image Proc. 1:97-106 (1972).

6. Scudder, H., Proc. IEEE 66:628-637 (1978).

7. Kak, A., Proc. IEEE 67:1245-1272 (1979).

8. Nolan, P., Brown, D., and Rothwell, E., Fourteenth Symposium (International) on Combustion, The Combustion Institute, Pittsburgh, 1973, pp. 1143-1150.

9. Chu, T., Gill, W., Moore, J., Hobbs, M., Gritzo, L., and
Moya, J., 41st International SAMPE Symposium and Exhibition, Covina, CA 1996.

10. Crawford, C., and Kak, A., Ultrasonic Imaging 4:234266 (1982).

11. Schomberg, H., Beil, W., McKinnon, G., Proksa, R., and Tschendel, O., Acta Electronica 26:121-128 (1984).

12. Mason, W., Ultrasonics Symposium Proceedings, 1976, pp. 610-617.

13. Generazio, E., and Roth, D., J. Am. J. Ceram. Soc. 72:1282-1128 (1989).

14. Lynnworth, L., Papadakis, E., and Fowler, K., Intl. Adv. Nondestruct. Test. 5:71-115 (1977).

15. Norton, S., and Linzer, M., Ultrasonic Imaging 4:201233 (1982).

16. Biot, M. A., J. Acous. Soc. Amer. 28:168-178 (1956)

17. Biot, M. A., J. Acous. Soc. Amer. 28:179-191 (1956).

18. Biot, M. A., J. Appl. Phys. 33:1482-1498 (1962).

19. Kuttruff, H., Ultrasonics: Fundamentals and Applications, Elsevier, New York, 1991, p. 207.

20. Leclaire, P., Kelders, L., Lauriks, W., Gloriuex, C., and Thoen, J., J. Acous. Soc. Amer. 99:1944-1948 (1996).

21. Henry, M., Lemarinier, P., Allard, J., Bonardet, J., and Gedeon, A., J. Appl. Phys. 77:17-20 (1995).

22. Allard, J., Castagnede, B., Henry, M., and Lauriks, W., Rev. Sci. Instrum. 65:754-755 (1994).

23. Kundu, P. K., Fluid Mechanics. Academic Press, San Diego, 1990, pp. 284-287.

24. Bacri, J., Hoyos, M., Rakotomalala, N., Salin, D., Bourlin, M., Daccord, G., Lenormand, R., and Soucemarianadin, S., J. Physique III France 1:1455-1466 (1991).

25. Johnson, D., Koplik, J., and Dashen, R., J. Fluid Mech. 176:379-402 (1987).

26. Herzfeld, K., and Litovitz, T., Absorption and Dispersion of Ultrasonic Waves, Academic Press, New York, 1959.

27. Bhatia, A., Ultrasonic Absorption, Oxford University Press, New York, 1967.

28. Yuan, Z., Fall Meeting, Western States Section, The Combustion Institute, Palo Alto, CA 1995.

29. Hughey, B., and Santavicca, D., Combust. Sci. Technol., 29:167-190 (1982)

30. Tse, S. D., (1996) Ph.D. thesis, University of California, Berkeley.

31. Tse, S. D., and Fernandez-Pello, A. C., Transport Phenomena in Combustion: Proceedings of the Eighth International Symposium on Transport Phenomena in Combustion, Vol. 1, S. H. Chan, Ed.), Taylor \& Francis, San Francisco, CA 1996, pp. 689-700.

Received 23 October 1997; accepted 6 April 1998 